\title{
Labyrinthe
}

$23 \mid 2006$ (1)

Quatre chercheurs de l'étranger

\section{Ludger Schwarte : l'esthétique performative du droit}

Guillaume Paugam et Charles Ruelle

\section{(2) OpenEdition}

1 Journals

Édition électronique

URL : http://journals.openedition.org/labyrinthe/1155

DOI : $10.4000 /$ labyrinthe.1155

ISSN : 1950-6031

Éditeur

Hermann

Édition imprimée

Date de publication : 15 janvier 2006

Pagination : 27-30

ISBN : 2-9526131-0-9

Référence électronique

Guillaume Paugam et Charles Ruelle, « Ludger Schwarte : I'esthétique performative du droit »,

Labyrinthe [En ligne], 23 | 2006 (1), mis en ligne le 23 juillet 2008, consulté le 05 mai 2019. URL : http:// journals.openedition.org/labyrinthe/1155; DOI : 10.4000/labyrinthe.1155

Propriété intellectuelle 


\title{
Ludger Schwarte : l'esthétique performative du droit
}

\author{
Guillaume PAUGAM et Charles RuElle
}

\begin{abstract}
"Toute manifestation de la vie de l'esprit humain peut être conçue comme une sorte de langage, et, à l'instar de toute vraie méthode, cette conception a pour effet de poser partout les problèmes d'une façon nouvelle. On peut parler d'un langage de la musique et de la sculpture, d'un langage de la justice, qui n'a directement rien à voir avec la langue dans laquelle sont rédigées les sentences des tribunaux allemands ou anglais; on peut parler d'un langage de la technique, lequel n'est point le langage professionnel des techniciens ${ }^{1}$. »
\end{abstract}

Il est difficile de se représenter autrement le geste de Ludger Schwarte qu'au regard de la célèbre injonction que Walter Benjamin lançait à son ami Scholem dans cette lettre de 1916, restée inédite de son vivant mais source d'inspiration constante pour son auteur au moins jusqu'au début des années 1930. Il fallait prendre très au sérieux le défi qui y est exprimé, se décider à examiner patiemment tous les présupposés langagiers et non seulement linguistiques - d'une certaine mise en scène du droit pour que la familière justice apparaisse effectivement « d'une façon nouvelle ». C'est ce renversement de perspective qui, dans l'extrait de « Die Inszenierung von Recht...» que nous avons choisi, amène Schwarte à reconsidérer le processus judiciaire selon ses règles énonciatives, dans ses petits arrangements avec la légalité, dans sa mise en scène.

Dans son titre et, plus encore, dans son écriture, l'article de Schwarte fait d'abord pendant à La Mise en scène de la vie quotidienne de Goffman et à sa présentation minutieuse du monde comme jeu social ${ }^{2}$. Si le monde

\footnotetext{
1. «Sur le langage en général et sur le langage humain » [trad. Maurice de Gandillac, revue par Rainer Rochlitz], dans Walter Benjamin, Euvres I, Paris, Gallimard, coll. « folio essais », 2000, p. 142.

2. Erving Goffman, La Mise en scène de la vie quotidienne, 1. La Présentation de soi, 2. Les Relations en public, [The Presentation of Self in Everyday Life, trad. de l'anglais par A. Accardo; Relations in Public, trad. de l'anglais par A. Kihm], Paris, Les Éditions de Minuit, coll. « Le sens commun », 1973.
} 
de Goffman est un théâtre de règles systématiquement décrites du point de vue de l'acteur, la scène judiciaire de Schwarte présente plutôt la « face » d'une dramaturgie expérimentale où c'est le cas qui peut déterminer la régularité. La mise en scène et les codes de procédures servent quant à eux l'illusion de la justice et de la prise en compte des acteurs - qui se trouvent exister uniquement au moment du verdict. Le déroulement ordonné du procès dissimule en fait l'absence véritable de règles et l'anarchie dont il procède. La déconstruction de l'arène judiciaire, en répondant à distance à l' «Öffentlichkeit» habermassienne, permet ainsi d'exhiber les présupposés rituels, juridiques et scéniques, qui font du procès un lieu de production du vrai, et justifie l'ambition d'une véritable « théorie performative du droit ${ }^{3} »$.

Cette dernière - et c'est proprement remarquable - ne focalise pas uniquement son attention sur les énoncés du discours juridique, selon un point de vue naïf et pseudo-analytique ${ }^{4}$. Elle ne réduit pas non plus la justice à l'appareil totalitaire d'un potentat légal - le porteur du skeptron selon Bourdieu et, plus récemment, celui qui décrète l' «état d'exception» selon Agamben -, mais tente de circonscrire la scène complexe d'une dramaturgie à la signification beaucoup plus profonde, et plus hégélienne, où l'individuel représente le droit contre la loi. En effet, selon Schwarte,

la performance n'est pas extérieure au droit. Mais la performance ne concerne pas seulement la déclaration et son acception. Le droit n'est pas un simple mode d'emploi, ni même une description de la réalité, mais une interaction sociale 5 .

À l'origine de cette théorie performative du droit, Schwarte présente deux résultats: d'une part, celui de la double dimension, dramaturgique et juridique, des procès criminels et l'inscription, qui en découle, de «l'exercice du droit dans le champ des pratiques culturelles et des mises

\footnotetext{
3. Ludger Schwarte, «Angemessenes Unrecht - Gerechter Zufall : Modelle rechtlicher Performanz », [article à paraître en 2006 dans Paragrana, Internationale Zeitschrift für Historische Anthropologie, numéro sur le thème « Materialität des Rechts »].

4. Austin n'échappe-t-il pas lui-même à ce point de vue naïf quand il affirme que tous les actes performatifs ne valent que selon les termes d'un protocole commun aux locuteurs? Voir J. L., Austin, Quand dire, c'est faire [trad. de l'anglais par G. Lane], Paris, Seuil, coll. « Point », p. 49, 79 et 91 pour tout ce qui concerne les «procédures», « conventions » et « conditions » en tant que conditions de félicité des performatifs.

5. «Angemessenes Unrecht - Gerechter Zufall...», op. cit.
} 


\section{Ludger Schwarte: l'esthétique performative du droit}

en scène politiques ${ }^{6} »$. Bien présent dans cet article, le rapport du droit au théâtre rencontre une autre analyse fondatrice: la spécificité de la relation du droit au corps des acteurs judiciaires dans nos sociétés contemporaines dites démocratiques, par rapport au droit antique - grec et romain - et au droit moderne - notamment hégélien - vis-à-vis desquels il apparaît « historiquement et culturellement hétérogène ${ }^{7} »$. Alors que le tribunal populaire des Grecs supposait l'identité des corps ${ }^{8}$, conçus négativement dans une opposition à l'esprit, et que Hegel persistait à voir ces derniers comme des organismes dominés par leur détermination physiologique, la législation actuelle doit s'accommoder de nouveaux objets (embryons, gènes, spermatozoïdes, les «actants » de Bruno Latour ${ }^{9}$, etc.) sur lesquels elle agit mais dont elle doit virtualiser la corporéité pour rendre crédible son action.

Dans ce cadre, le système représentatif se trouve lui-même justifié, mais révèle, en d'autres circonstances, la négation du corps des acteurs représentés lors du procès: cela, d'une part, en raison de l'impossibilité que les représentés ont de s'exprimer et, d'autre part, en raison de la nature même du procès qui assigne à chacun un rôle objectivé par la présence du public, qui plus est seulement au moment du verdict, quand l'accusé se trouve réellement reconnu coupable.

D'où, chez Schwarte, une analyse qui se déploie ici à partir de la définition des «acteurs» (3) et des modalités de distribution de la parole (3.1).

6. Ludger Schwarte, art. cit., op. cit.; voir aussi sur ce point précis Gertrud Koch, Sylvia Sasse, Ludger Schwarte, (éd.), Kunst als Strafe, Zur Ästhetik der Disziplinierung, Munich, Wilhelm Fink, 2003, notamment l'article de Ludger Schwarte, «Katharsis und Kunstlosigkeit: von der Publikumsherrschaft zur Anarchie der Kunst », p. 25-63.

7. Ludger Schwarte, « Angemessenes Unrecht - Gerechter Zufall », op. cit.

8. Sur ce point, l'analyse de Ludger Schwarte se déploie à partir du modèle institutionnel tel qu'il a cours au IV et $\mathrm{V}^{\mathrm{e}}$ siècle à Athènes et dont Aristote s'est notamment fait l'écho dans Les Politiques. De là ressort que pour Schwarte, seul le tribunal athénien est véritablement démocratique, contrairement à notre système juridique. Dans le tribunal athénien, «tout citoyen est juge, le juge est plusieurs corps ». Il représente « plusieurs manières de ressentir le droit, ne fait pas qu'abattre une règle mais intègre le hasard et une dimension véritablement publique à sa décision». Voir « Angemessenes Unrecht - Gerechter Zufall », op. cit., ainsi que « Inszenierung von Recht - Der unbekannte Körper in der demokratischen Entscheidung », p. 94 sqq.

9. «Puisque les humains doués de parole aussi bien que les non-humains muets ont des porte-parole [...], je propose d'appeler actants tous ceux, humains ou non-humains, qui sont représentés afin d'éviter le mot d'acteur trop anthropomorphique», dans Bruno Latour, La Science en action. Introduction à la sociologie des sciences, Paris, La Découverte, 2005 [trad. de l'anglais par Michel Biezunski], p. 202. Schwarte, dont une partie des travaux porte, comme Latour, sur la vie de laboratoire et ses mises en scène raffine la terminologie de ce dernier en parlant de «biones » (Bioten) pour les actants organiques. Voir sur ce point «Die Inszenierung von Recht... », op. cit., p. 117. 
L'appareil judiciaire apparait dans sa fonction rituelle, quasi religieuse, qui induit un processus de subjectivation propre: le «nous » d'une universalité artefactuelle (3.2) dont l'introduction des objets (3.3) et des corps (4) assure la réification - parce que le délit porte sur les « biens », parce que le conflit implique des personnes, il est donc de reconnaissance. Celle-ci passe par le langage qui noue le drame pratique et esthétique des scènes judiciaire et théâtrale (4.1). Chez Schwarte, le traitement du langage et de la discussion vient toutefois radicaliser la conception de Jürgen Habermas ${ }^{10}$ pour faire porter la valeur du droit sur le terme ultime du procès, à savoir le jugement, plutôt que sur le processus discursif organisé par la procédure.

L'analyse du spectacle de la justice en tant que scène alternative du droit fait quant à elle proprement l'objet de la dernière partie de l'exposé de Schwarte. L'examen de ses conditions légales d'invalidité (et donc aussi de validité) (4.2), d'ouverture à son propre dehors, aboutit à l'idée que c'est de l'événement qu'il faut faire surgir la règle (4.3). On pense ici fortement à Agamben, mais Schwarte n'érige cependant pas l'anarchie en modèle ni l'exception en état sublimé de la loi. À charge pour celui qui juge, dans le geste exactement inverse de celui du législateur, de dématérialiser le corps du justiciable afin de gérer l'inscription judiciaire de la loi par le procès public de ses exceptions (5 et 5.1). Schwarte, ayant traqué ainsi dans l'illusion de la procédure la contingence de la rationalité du droit, exhibe toute la violence et l'autocratisme de la décision judiciaire. Reste à trouver de quoi transformer les pratiques actuelles de nos institutions pour en refonder le caractère véritablement démocratique et assurer aux citoyens la légitimité de la sentence prononcée en leur nom.

10. Pour le dire simplement, chez Habermas la validité des énoncés pratiques - et la légitimité juridique - s'établit dans le cadre d'une décision argumentée au sein d'un contexte public. Pour Schwarte, la discussion a une dimension relativement formelle qui apporte peu à l'autonomie du jugement et ne le rend pas dépendant d'une procédure discursive d'où il tirerait son caractère valide ou légitime, d'où l'anarchie. La « légitimation par la procédure », dont parlait Luhmann, devient soudainement un masque à la validité du procès, plutôt que son fondement véritable. 